\title{
A system for archiving and reporting environmental radioactivity monitoring data
}

\author{
P. McGinnity, L. Currivan, P. Fitzgerald and D. Pollard \\ Radiological Protection Institute of Ireland, 3 Clonskeagh Square, Dublin 14, Ireland
}

\begin{abstract}
A new information system for archiving and reporting the RPII's environmental radioactivity monitoring data has been designed. The main objectives of the proposed system are: centralised storage of these data in a standard format; and standardised reporting and exporting functionality. This paper presents a conceptual model and prototype of the proposed system.
\end{abstract}

\section{INTRODUCTION}

The Radiological Protection Institute of Ireland (RPII) carries out environmental monitoring in Ireland to ensure that environmental radiation remains within internationally agreed and legal safety limits [1]. The key elements of the RPII monitoring programme are the assessment of ambient radioactivity based on measurements of radioactivity in air and of external gamma dose rate from a network of permanent monitoring stations located throughout the country; assessment of levels of radioactivity in a variety of food products and drinking water; and assessment of levels of radioactivity in the Irish marine environment based on sampling and measurement of seawater, sediment, seaweed, fish and shellfish. The programme combines round-the-clock measurements from the permanent monitoring network and a programme of sampling followed by laboratory testing. This continuous testing is complemented by project-based monitoring to investigate particular aspects of radioactivity in the environment.

The results of the monitoring programme are used to support the RPII's advisory, information provision and emergency management roles through publication of a range of reports, advice notes and public information material, notably an annual environmental monitory report [2] and updates to the RPII's website. Data are also used to fulfil international commitments including the European Commission's (EC) EURDEP (EUropean Radiological Data Exchange Platform) [3] and REM (Radioactivity Environmental Monitoring) [4] databases; and the Oslo Paris Conventions' (OSPAR) Radioactive Substances Committee (RSC) [5].

The current data flow of the monitoring programme is demonstrated in the top and bottom sectors of Figure 1.

\section{NEW INFORMATION SYSTEM FOR MONITORING DATA}

Currently a number of issues restrict efficient analysis, interpretation and publication of the RPII's monitoring data. These issues include:

- Data are stored in two separate operational databases, laboratory measurements in a Laboratory Information Management System (LIMS) and gamma dose rate data in a Permanent Monitoring System (PMS). Each has a different format resulting in (limited) reporting and exporting mechanisms.

- Charts and tables for publications are prepared manually which involves extensive rekeying of data. Data therefore have to be checked every time they are used, an inefficient use of personnel time. 


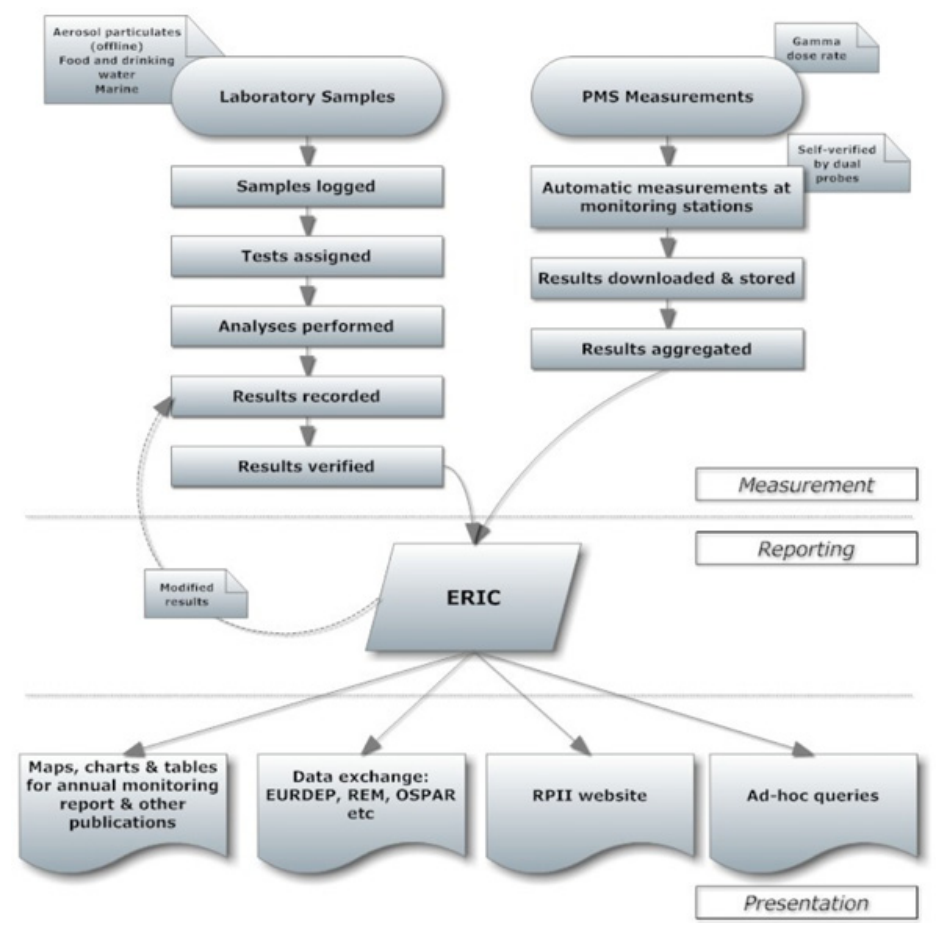

Figure 1. Data flow in the RPII monitoring programme.

- Despite the highly spatial nature of the data - all measurements are associated with a sampling location - geographic information is used only to a limited extent.

- Metadata is limited and auxiliary data (e.g. sampling plans, logs and internal project reports) are not linked or stored with the data.

- The RPII's website includes a module to display monitoring locations on Google maps hyperlinked to the latest measurements. However there is no link to LIMS. Rather all data, with the exception of gamma dose rate measurements, are updated manually.

To address these issues a new information system, provisionally titled ERIC (Environmental Radioactivity Ireland Central), for improved archiving and reporting of the RPII's monitoring data has been designed. The two primary aims of ERIC are:

- Centralised storage of these data in a standard format; and

- Standardised reporting and exporting functionality.

The move to develop the system has been influenced by other similar national systems such as the German IMIS (Integrated Measurement and Information System) [6] and the public-facing Réseau National in France [7] as well as international systems developed by the EC [3, 4] and IAEA [8].

The basic architecture of ERIC comprises a relational database containing measurements and locations and a geodatabase for spatial data. A range of SQL (Structured Query Language) queries are required both for populating the database, i.e. transferring updated data from LIMS and PMS, and for reporting and exporting.

A prototype system has been developed as a proof of concept; to demonstrate the advantages of such as system; and to assist in working through a number of expected issues. The prototype database has been based on the EC's REM database [4], with certain extensions. Measurements imported into the prototype thus far include those from a three year study of radioactivity in groundwater drinking water 


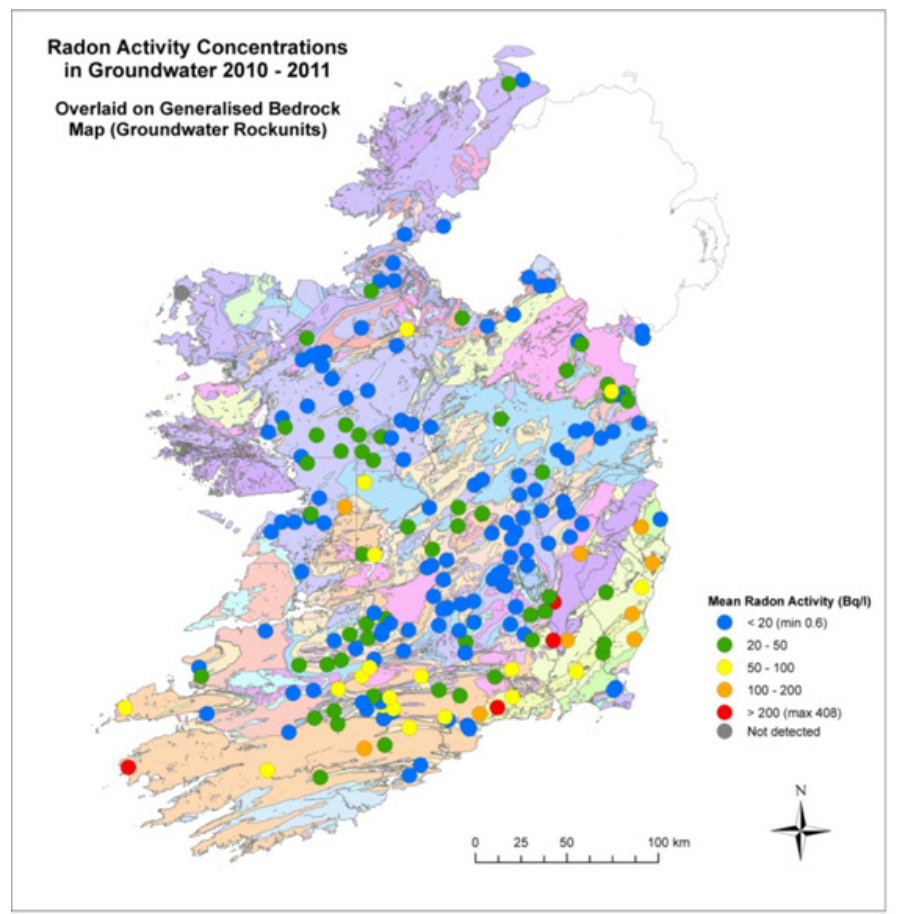

Figure 2. Sample map showing average activity concentration of radon in groundwater overlaid on bedrock type. Source: GSI, RPII.

sources which is nearing completion and the results of additional monitoring performed by the RPII in response to the accident at the Fukushima Daichi nuclear power plant in Japan.

The incorporation of ERIC into the monitoring programme's data flow is presented in Figure 1. Note that only verified data from LIMS is imported. This ensures that users can confidently use and publish the data without further checking.

The system employs Geographic Information System (GIS) software which offers two major benefits. Firstly, GIS facilitates the use of the map as a 'window' [9] onto the monitoring data offering a huge improvement in the visualisation of data. GIS also enables data of any type to be linked by the location to which they pertain. In ERIC, this functionality offers the potential for data from different sources, projects, measurement times or sample types to be presented and analysed as a single dataset, hence creating synergies and assisting more efficient identification of both temporal and geographical trends. As a simple example, Figure 2 presents average activity concentration of radon in groundwater overlaid on bedrock data prepared by the Geological Survey of Ireland (GSI), a first step in assessing any correlation between the two parameters.

The use of GIS also provides immediate access to a range of standard functionality including:

- Mapping of all relevant monitoring points for user selected sample types

- Temporal queries: displaying data for one or more sample type for a user-selected time window

- Location queries: clicking through monitoring location to access data (e.g. activity concentrations, doses and other related documentation) relevant to that location.

- Spatial queries: various reports (e.g. time-series, interpolated values etc) generated for monitoring results from within a user-defined area of the map.

- Standard GIS tools for manipulating the map including zooming, panning, calculation of straight line distances and basic spatial analysis. 


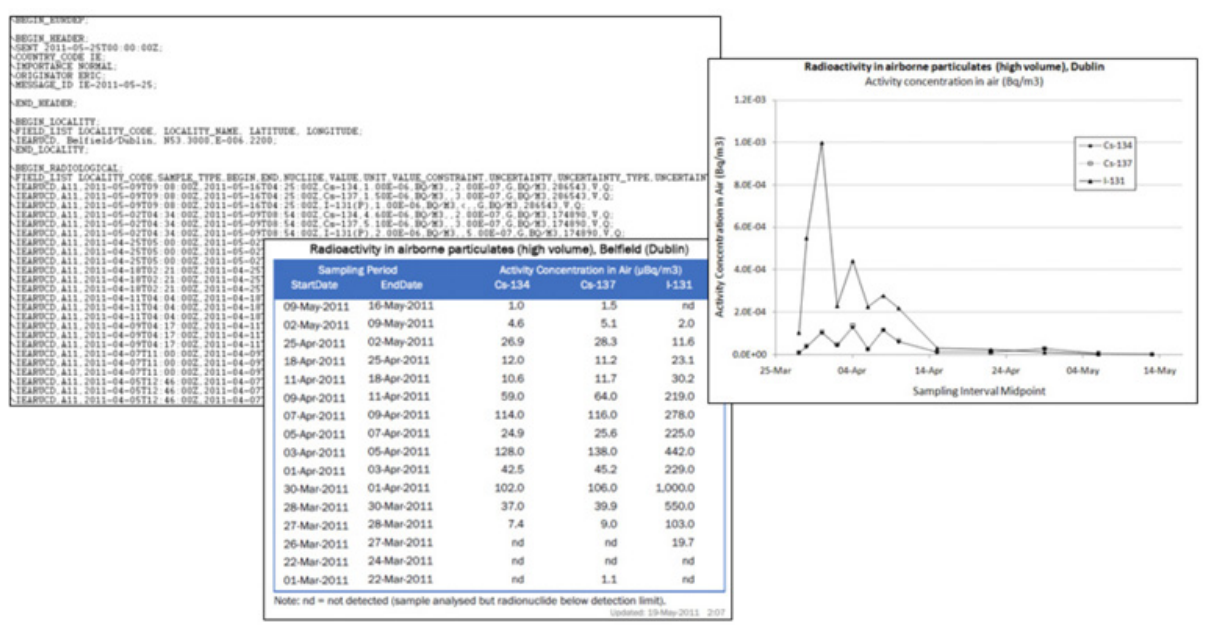

Figure 3. A range of outputs from a single high volume airborne particulate dataset.

SAP Crystal Reports is used in ERIC to publish and export data in tabular and graphical formats. Figure 3 presents the same selection of data - activity concentrations in aerosol particulates from a high volume sample measured in response to the Fukushima accident from March to May 2011 - published in various formats.

\section{CONCLUSIONS}

The developments of the conceptual model and prototype of ERIC have demonstrated the high potential for such an information system in RPII. The visual impact of data is improved which can only help the RPII in performing its information provision and advisory roles. Results can be issued as soon as they have been verified to ensure they remain timely. The efficiency with which data can be published in a variety of formats, notably without further checking once the queries used have been comprehensively tested, frees up valuable manpower to undertake other projects. With specific regard to the RPII's emergency management role, response the Fukushima incident involving additional monitoring of certain sample types for public reassurance highlighted the need for all of the above. The use of GIS also opens access to wide range of user functionality for querying and interpreting data directly from the map. As noted by Grimshaw [10], further intangible benefits undoubtedly will become apparent once the data has been geocoded and displayed on a map.

The prototype has been useful in identifying and resolving various issues which will be encountered more generally in real system. These include:

- treatment of minimum detectable activities in maps and charts;

- consistent aggregation of data;

- maintaining consistency with source databases;

- optimal presentation of data on maps considering colours, classification systems, background imagery etc; and

- maintaining flexibility in reports for different formats and target audiences while keeping their number manageable.

ERIC also serves to put in place the basic GIS and reporting infrastructure required for a range of possible future uses in the RPII. Examples include monitoring project planning and the development of sampling strategies; and quantitative comparison of measurements with model results e.g. atmospheric and aquatic dispersion modelling. The system could also serve as the basis of a future web GIS which 
could enable direct interrogation of the RPII's monitoring data by the public. The benefit of GIS in linking disparate data sources by measurement location could also assist in contextualising RPII through comparison with similar measurements performed elsewhere and in encouraging collaboration both within Ireland and beyond.

In Europe, the European Community INSPIRE directive [11] has established an infrastructure for spatial information to support Community environmental policies, and policies or activities which may have an impact on the environment. Under the terms of the directive there is a requirement on all public bodies holding any spatial information principally related to the environment to advertise the fact that they have data and its origins (metadata) (or that the data is available from them). ERIC has been initiated to assist the RPII, as a public body, in complying with INSPIRE requirements. Four sets of Implementing Rules provided by the Directive setting out how the various elements of the system (metadata, interfaces, network services, access conditions, etc) will operate have been consulted in the development.

It is also envisaged that ERIC will play a data conservation role. The RPII and other organisations have been performing monitoring of environmental radioactivity in Ireland for many years. Older data which are required for assessing long term trends and establishing baselines are stored in legacy databases or paper-based records. Many data are therefore inaccessible or have already been lost. By importing these data and supporting documentation into ERIC and presenting them in the same format as current measurements they are not only preserved but can only add to the RPII's institutional memory.

\section{References}

[1] McGinnity P., Currivan L., and Pollard, D., 2011. A Peer Review of the RPII Environmental Monitoring Programme, 2009. Submitted ICRER 2011, Hamilton, Canada.

[2] McGinnity P., Currivan L., Dowdall, A., Fegan, M., Hanley, O., Hayden, E., Kelleher, K., McKittrick L., Somerville, S., Wong, J. and Pollard, D., 2010. Radioactivity Monitoring of the Irish Environment 2009. RPII-10/02. Radiological Protection Institute of Ireland, Dublin.

[3] De Vries G., De Cort M., Tanner V., 2005. EURDEP: A Standard Data-format and Network for Exchanging Radiological Monitoring Data. Conference Proceeding 2005 International Conference on Monitoring, Assessments and Uncertainties for Nuclear and Radiological Emergency Response; 21st - 25th November 2005, Rio de Janeiro, Brazil.

[4] European Commission, 2009. Environmental radioactivity in the European Community, 20042006. EUR 23950 EN. Luxembourg: European Commission.

[5] OSPAR Commission, 2009. Towards the Radioactive Substances Strategy objectives Third Periodic Evaluation. [Internet] Available from <http://www.ospar.org/documents/dbase/ publications/p00455_3PE.pdf $>$ [Accessed 25 May 2010].

[6] BFS, 2011. Website: Das integrierte Mess- und Informationssystem (IMIS). [Internet] Available from < http://www.bfs.de/en/ion/imis/imis_uebersicht.html > [Accessed 25 May 2010].

[7] IRSN, ASN, 2011. Website: Réseau National de Mesures de la Radioactivité de l'Environnement. [Internet] Available from <http://www.mesure-radioactivite.fr/public > [Accessed 25 May 2010].

[8] IAEA, 2011. Website: MARiS: Marine Information System. [Internet] Available from $<\mathrm{http} / / /$ maris.iaea.org/> [Accessed 25 May 2010]

[9] Heywood, I., Cornelius, S. and Carver, S., 2006. Introduction to Geographical Information Systems (3rd edition). Harlow: Pearson.

[10] Grimshaw, D.J., 2000. Bringing Geographical Information Systems into Business (2nd edition). New York: John Wiley and Sons.

[11] European Commission, 2007. Directive 2007/2/EC of the European Parliament and of the Council of 14 March 2007 establishing an Infrastructure for Spatial Information in the European Community (INSPIRE). Official Journal of the European Union L 108/1. 